\title{
Early Symptom Development and Histological Changes Associated with Migration of Bursaphelenchus xylophilus in Seedling Tissues of Pinus thunbergii
}

\author{
Y. Ichihara, K. Fukuda, and K. Suzuki, Graduate School of Agricultural and Life Sciences, The University of \\ Tokyo, Bunkyo-ku, Tokyo 113-8657, Japan
}

\begin{abstract}
Ichihara, Y., Fukuda, K., and Suzuki, K. 2000. Early symptom development and histological changes associated with migration of Bursaphelenchus xylophilus in seedling tissues of Pinus thunbergii. Plant Dis. 84:675-680.

In order to clarify the mechanism of pine wilt caused by the pinewood nematode (PWN), Bursaphelenchus xylophilus, nematode migration in tissues and disease symptoms in Pinus thunbergii seedlings were investigated. One-year-old seedlings were inoculated with different pathogenic isolates of PWN under two different temperatures. At an early stage of symptom development, a virulent isolate of PWN multiplied in both bark and xylem and was distributed in cortical resin canals, cortical tissue, and xylem resin canals at $30^{\circ} \mathrm{C}$. Cell death and disease symptoms developed in both bark and xylem. The virulent isolate of PWN at $25^{\circ} \mathrm{C}$ and the avirulent isolate of $\mathrm{PWN}$ at $30^{\circ} \mathrm{C}$ were distributed mainly in cortical resin canals, but rarely in xylem resin canals and cortical tissue. Disease symptoms and cell death occurred in cortical resin canals and rarely occurred in other tissues. These results demonstrated that the virulent isolate of PWN at low temperature and avirulent nematodes could not easily migrate to xylem resin canals and cortical tissue. It was shown that cell death and early symptom development coincided with PWN migration and, therefore, PWN invasion induces cell death and early symptom development.
\end{abstract}

Additional keywords: Japanese black pine, pine wilt disease, pinewood nematode

Pine wilt caused by the pinewood nematode (PWN), Bursaphelenchus xylophilus (Steiner and Buhrer) Nickle, is the most serious forest disease in Japan and East Asia. The amount of dead trees is large, ranging from 0.76 to 1.01 million $\mathrm{m}^{3}$ in 1994 to 1998 in Japan. In the northern part of Honshu in Japan, pine wilt disease has now invaded an area where the disease did not occur previously.

Isolates of the PWN that differ in pathogenicity have been identified $(12,14)$. Virulent isolates migrate and multiply in inoculated pines and induce disease

Corresponding author: Y. Ichihara

E-mail: ichiyu@ffpri-thk.affrc.go.jp

Current address of Y. Ichihara: Laboratory of Forest Pathology, Tohoku Research Center, Forestry and Forest Products Research Institute, Nabeyashiki 72, Shimo-Kuriyagawa, Morioka, 020-0123, Japan; current address of K. Fukuda: Program of Biosphere Functions, Department of Natural Environmental Studies, Institute of Environmental Studies, Graduate School of Frontier Sciences, The University of Tokyo, Bunkyo-ku, Tokyo 113-8657, Japan.

This research was supported by the Research Fellowships of the Japan Society for the Promotion of Science for Young Scientists (No. 084685).

Accepted for publication 29 February 2000.

Publication no. D-2000-0403-03R

(C) 2000 The American Phytopathological Society symptoms, resulting in decreased water potential and tree death (20), except at low temperatures. Virulent isolates multiply actively at high temperatures $\left(25\right.$ to $\left.30^{\circ} \mathrm{C}\right)$ but poorly at low temperatures $\left(<20^{\circ} \mathrm{C}\right.$; $1,3,4,11,22)$. Avirulent isolates slowly induce restricted disease symptoms and kill pine trees at low incidence (14). Avirulent isolates disperse restrictedly in pine trees and multiply poorly in pine trees and on fungi $(2,5-7,9,13)$. Therefore, it is assumed that PWN dispersion and multiplication are key factors in the occurrence of disease symptoms and tree death.

PWN distribution in host tissue has been described in several publications $(16,18$, $19,21,23)$. However, the migratory route of PWN in pine trees is not fully understood and the distributions of virulent and avirulent isolates in pine tissue have not been compared at high and low temperatures. Furthermore, the relationship between nematode presence and the denaturation of pine tissue remains unclear. In this study, in order to clarify PWN migration and the nematode's effects on host tissue, the location of PWNs and the occurrence of denatured host tissue were investigated in Pinus thunbergii Parl. seedlings inoculated with different pathogenic isolates of B. xylophilus under two different temperatures.

\section{MATERIALS AND METHODS}

P. thunbergii seedlings (1 year old) from the nursery at the Tanashi experimental station of The University Forests, The University of Tokyo, were transplanted into pots. The heights of the seedlings ranged from 12 to $20 \mathrm{~cm}$. Current-year shoots of each seedling were cut off, leaving only the terminal shoot. All experimental treatments were continued in airconditioned greenhouses at high (day, $30^{\circ} \mathrm{C}$ and night, $25^{\circ} \mathrm{C}$ ) or low (day, $25^{\circ} \mathrm{C}$ and night, $20^{\circ} \mathrm{C}$ ) temperatures and $70 \%$ humidity with a day length of $16 \mathrm{~h}$.

A virulent isolate (S6-1) and an avirulent isolate (C14-5) of PWN were procured from the Forestry and Forest Products Research Institute, Ibaraki, Japan. Nematodes were reared on Botrytis cinerea Pers. grown in potato dextrose agar in petri dishes at $25^{\circ} \mathrm{C}$ for 10 days in darkness and extracted using the Baermann funnel method (27). Ten thousand nematodes were inoculated into wounds $(1 \mathrm{~cm}$ in length) made with a razor blade at the bases of the terminal shoots of the pine seedlings.

Three treatments were applied: inoculation with the virulent isolate of PWN under high temperature (VH treatment), inoculation with the virulent isolate of PWN under low temperature (VL treatment), and inoculation with the avirulent isolate of PWN under high temperature (AH treatment). The number of inoculated seedlings was 16,12 , and 14 for the VH, VL, and AH treatments, respectively. Three seedlings from each treatment were harvested at just above the soil surface at 3 and 7 days after inoculation to determine PWN distribution. Shoots from these seedlings were cut $2 \mathrm{~cm}$ above and below the point of inoculation and PWNs were extracted from the bark and xylem separately, using the Baermann funnel method, and counted under a stereomicroscope.

To observe disease symptoms and nematode distribution in pine tissue, another three samples from each treatment were collected at 3 and 7 days after inoculation. The roots of these seedlings were washed and immersed in a $0.05 \%$ solution of acid fuchsin for several hours (26); then the shoots from these seedlings were cut $1.5 \mathrm{~cm}$ above and below the inoculation site and disease symptoms on the cut surfaces were examined. The area of bark discoloration and cavitation (cessation of water conduction) that was indicated by the xylem not being stained by acid fuchsin was recorded under a stereomicroscope. 
After the examination of the cut surfaces, samples were fixed in FAA $(37 \%$ formalin, $99.7 \%$ acetic acid, 95\% ethanol, and water at 2:1:7:10 vol $/ \mathrm{vol}$ ). All of the tissue from one seedling in each treatment from the fixed samples was washed and mounted in OCT compound (Miles Co., Elkhart, IN) in a Cryostat CM1500 cryomicrotome (Leica Instruments, $\mathrm{GmbH}$, Nussloch) and sectioned at $40-\mu \mathrm{m}$ thickness. When samples were sectioned, the OCT compound was applied to the cut surface and frozen to prevent the movement of cut fragments of PWN. All cut sections were put on glass slides and mounted in glycerin with added acid fuch- sin solution (95\% glycerin and $1 \%$ acid fuchsin at 10:1 g) to stain the cut fragments of PWN. All sections were observed under an Olympus BH-2 microscope and the cut fragments of PWN were counted in each tissue section.

In order to determine occurrence of disease symptom and cell death in pine seedlings, one seedling was collected from the $\mathrm{VH}$ treatment at $1,3,5$, and 7 days and from the $\mathrm{AH}$ treatment at 3 and 7 days after inoculation. These seedlings were stained with acid fuchsin as described above, and shoot sections were cut $1 \mathrm{~cm}$ above and below the inoculation site. Disease symptoms on the cut surfaces were observed as described above. Cut samples were fixed in $7.4 \%$ formalin in $50 \mathrm{mM}$ sodium phosphate buffer $(\mathrm{pH} 7.0)$. After washing, all samples were mounted in OCT compound and sectioned at $40-\mu \mathrm{m}$ thickness. Sections were rinsed in water and stained using the DAPI method (10) to detect the distribution of dead cells in the samples. The dead area in each tissue type of the sections was recorded.

\section{RESULTS AND DISCUSSION}

The largest number of PWNs in all treatments was extracted from pine tissue at the inoculation site, and the number gradually decreased away from the inocu-
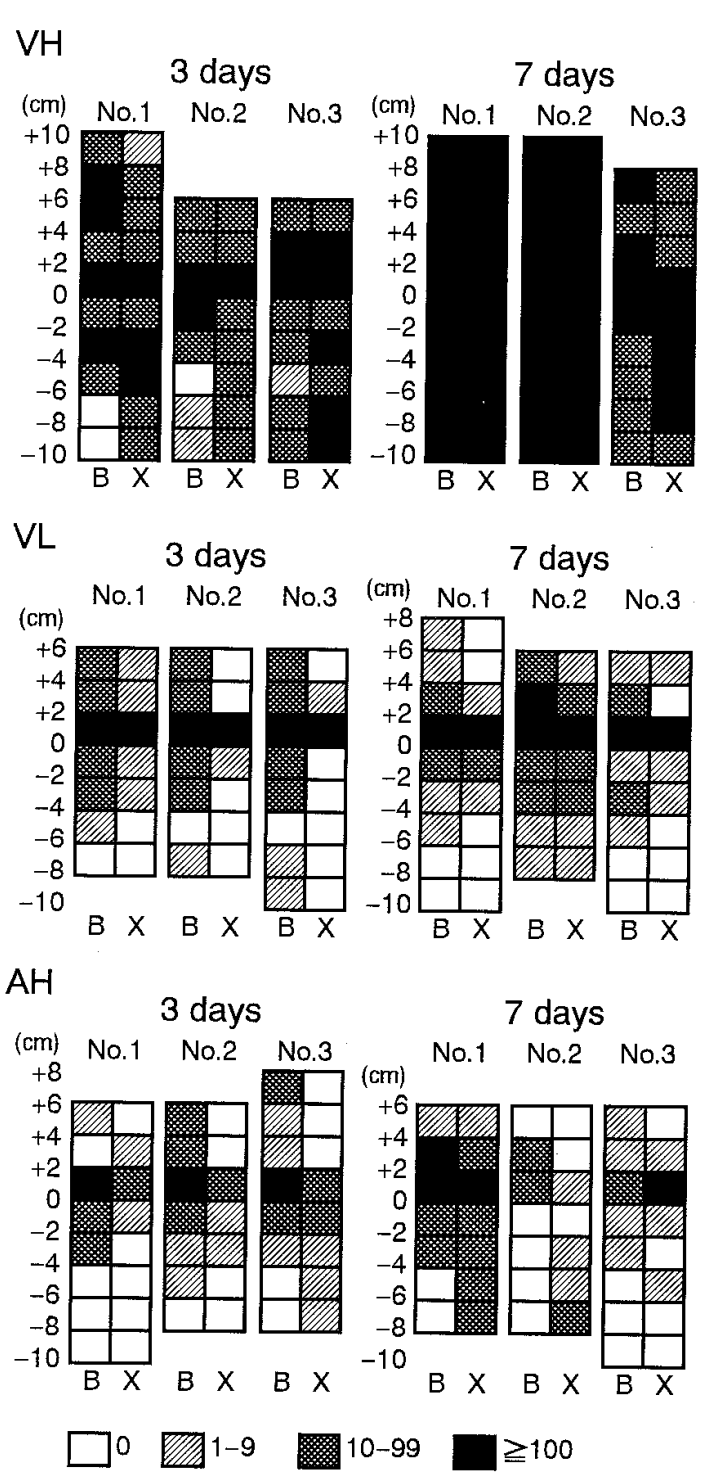

Fig. 1. Distribution of Bursaphelenchus xylophilus detected by extraction of pinewood nematodes (PWNs) in Pinus thunbergii seedlings inoculated with a virulent isolate of $\mathrm{PWN}$ at high temperatures $\left(30\right.$ to $\left.25^{\circ} \mathrm{C} ; \mathrm{VH}\right)$, a virulent isolate of $\mathrm{PWN}$ at low temperatures $\left(25\right.$ to $20^{\circ} \mathrm{C}$; VL), and an avirulent isolate of PWN at high temperatures $(\mathrm{AH})$ at 3 and 7 days after inoculation. Numbers above columns indicate seedling number. Each column indicates number of extracted PWN from bark (B) and xylem (X) tissues. Numbers at left side show the distance above (+) and below (-) the inoculation site in the stem of the seedling.
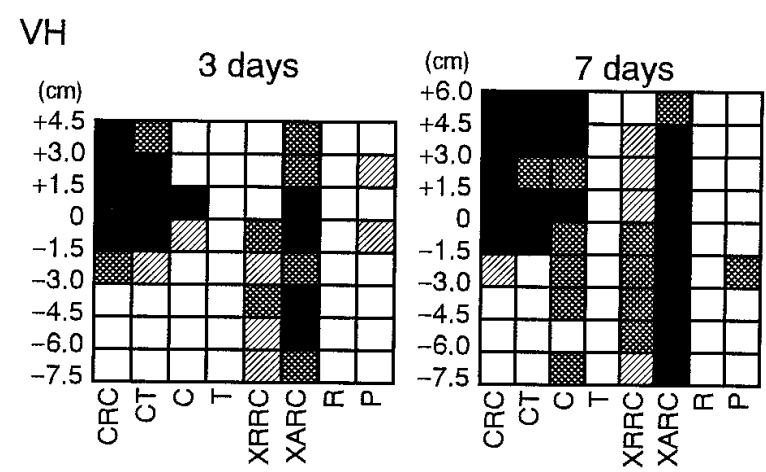

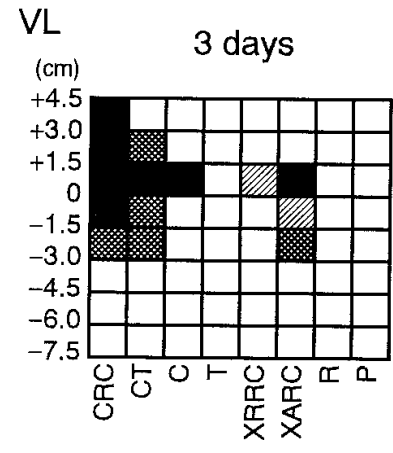

$\mathrm{AH}$
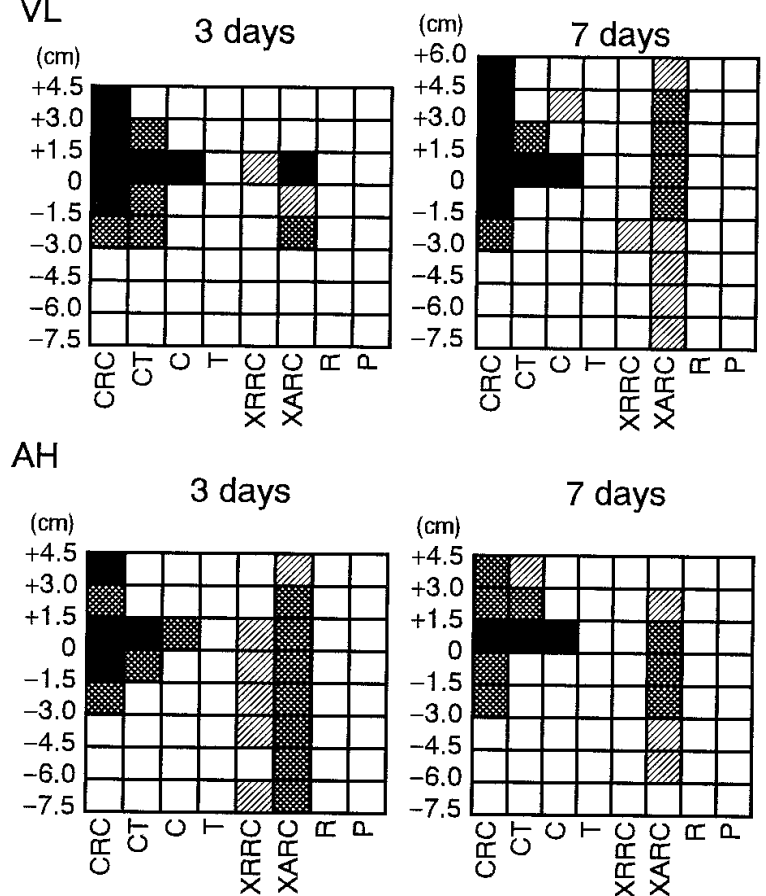

7 days
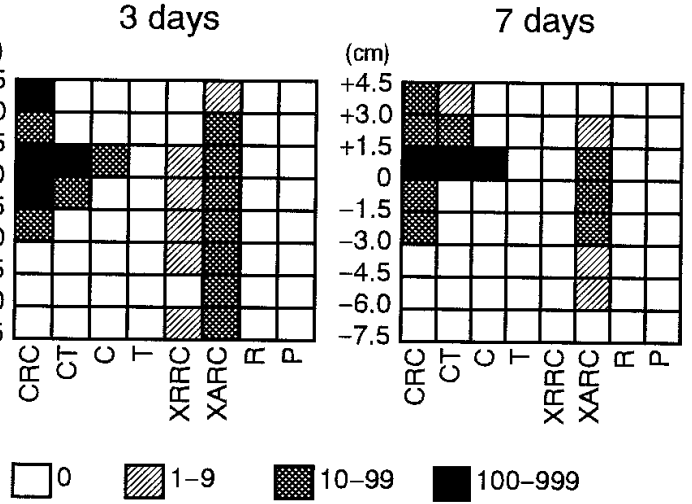

$100-999$
Fig. 2. Distribution of Bursaphelenchus xylophilus detected by anatomical observation in tissues of Pinus thunbergii seedlings inoculated with a virulent isolate of pinewood nematode $(\mathrm{PWN})$ at high temperatures (30 to $\left.25^{\circ} \mathrm{C} ; \mathrm{VH}\right)$, a virulent isolate of PWN at low temperatures ( 25 to $20^{\circ} \mathrm{C}$; VL), and an avirulent isolate of PWN at high temperatures (AH) at 3 and 7 days after inoculation. One set of columns indicates one seedling. Each column indicates number of cut fragments of PWN in cortical resin canals (CRC), cortical tissue $(\mathrm{CT})$, cambial zone $(\mathrm{C})$, tracheid $(\mathrm{T})$, radial resin canal in xylem (XRRC), axial resin canal in xylem (XARC), ray (R), and pith (P). Numbers at left side show the distance above (+) and below (-) the inoculation site in the stem of the seedling. 
lation site (Fig. 1). The PWNs were distributed throughout the tree in bark and xylem in all samples within 3 days after inoculation with the virulent isolate of PWN at high temperature (VH). The PWN number increased within 7 days after inoculation in the VH treatment. In the VL and $\mathrm{AH}$ treatments, PWNs were distributed in the bark 3 days after inoculation but less distributed in the xylem. At 7 days after inoculation, PWNs were distributed mainly in the bark, but a small number of PWNs also were distributed in the xylem beyond the inoculation site.

Histological observation of tissue sections showed that cut fragments of PWN were distributed in cortical resin canals, cortical tissue, and axial and radial resin canals of the xylem in the VH treatment 3 days after inoculation (Figs. 2 and 3A and B). Very few PWNs were in the pith and were not observed in tracheids or ray parenchyma tissue. PWNs appeared to invade the cambial zone near the inoculation site (Fig. 3C). Large populations of the PWN often invaded the cortical tissue and destroyed cell walls. PWNs were not observed in bark more than $3 \mathrm{~cm}$ below the inoculation site, where cortical resin canals were absent. At 7 days after inoculation, many PWNs were distributed throughout the tree, mainly in cortical and xylem resin canals and in cortical tissue and the cambial zone, well beyond the site of inoculation. However, no nematodes were observed in the tracheids and rays, and only a few PWNs were observed in the pith near the inoculation site.

Radial resin canals pass from the xylem across the cambium, and their ends appear spherical in bark tissue (Fig. 3D). The spherical ends were not connected to cortical resin canals. PWNs were observed within radial resin canals, crossing the cambium (Fig. 3D), and in the spherical ends.

In previous studies, PWNs have been observed histologically in the axial and radial resin canals of the xylem, in the cortical resin canals, and in the cambial zone $(16,18,19,21,23)$. However, these observations did not quantify the presence of PWNs in host tissues. The histological observations in this study quantified the distribution of PWNs in pine tissues. PWNs apparently could not move in the bark of the lower parts of seedlings because there were no resin canals to serve as the pathway for PWN migration. PWNs also have been observed in the tracheids near the inoculation site or migrating from the rays to the tracheids $(18,21)$. However, in our study, no nematodes were observed in the tracheid and ray.

The route by which PWNs cross the cambium had not previously been clearly identified. Our study indicates that PWNs can cross cambium through radial resin canals without destroying the tissue in the cambial zone. However, it is thought that the PWN cannot migrate from cortical resin canals to xylem resin canals directly because the spherical ends of the radial resin canals are not connected with cortical resin canals. These observations suggest that, when the inoculation wounds penetrate the xylem, PWNs can directly enter axial or radial resin canals in the xylem; whereas, when the wound does not penetrate the xylem, PWNs may first enter the cortical resin canals and break cells around the cortical resin canals to invade the cortical tissue, then invade the ends of radial resin canals that connect with the xylem resin canals.

Histological observation in the VL and $\mathrm{AH}$ treatments showed that PWNs were
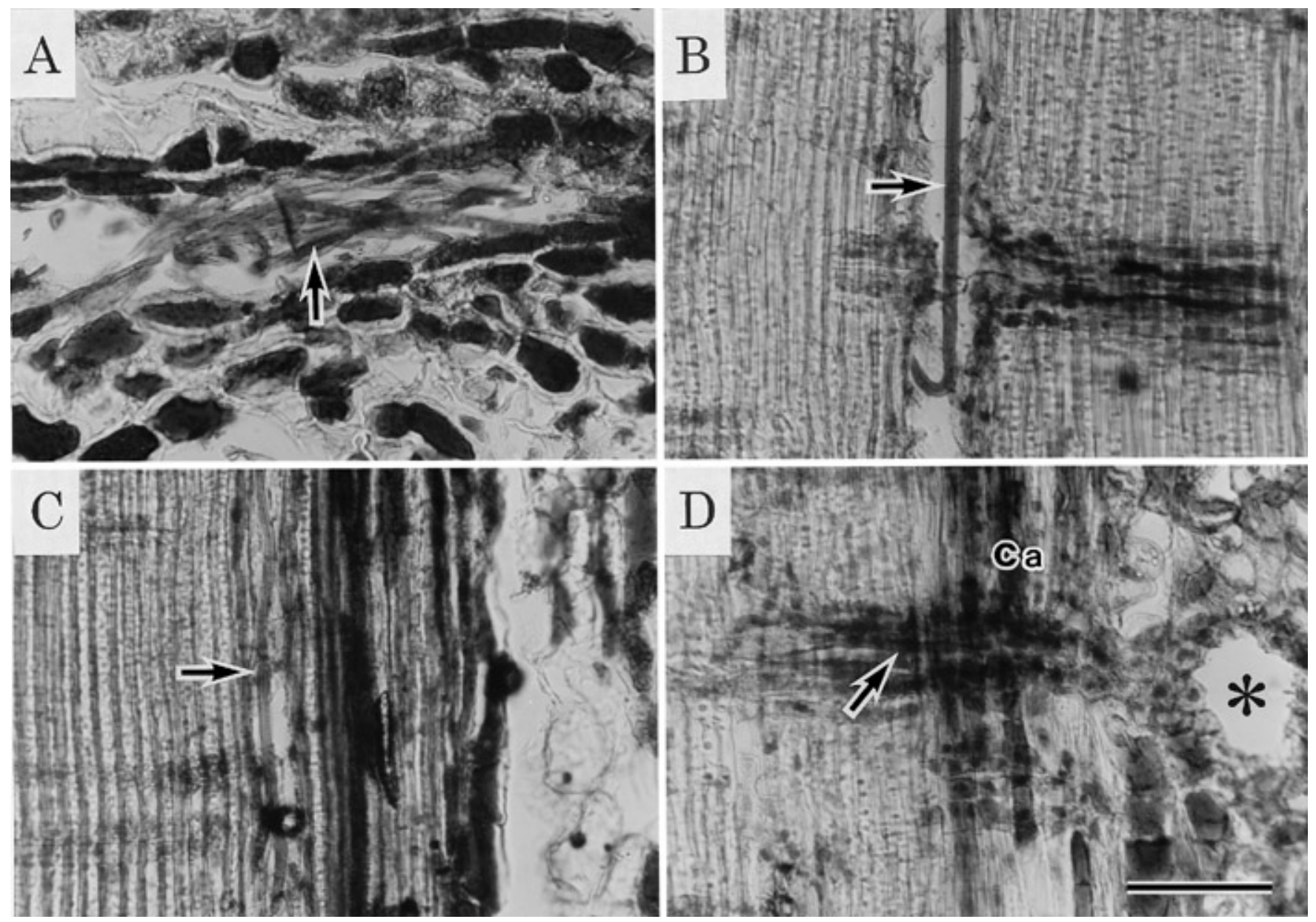

Fig. 3. Photomicrograph showing the distribution of nematodes (arrows) in Pinus thunbergii seedlings inoculated with a virulent isolate (S6-1) of Bursaphelenchus xylophilus. (A) Nematode colonization of a cortical resin canal. (B) Nematode within an axial resin canal of xylem. (C) Nematode in region of the cambium. (D) End of radial resin canal crossing the cambium. A nematode is present within the radial resin canal. $\mathrm{Ca}=\mathrm{cambium}, *=\mathrm{a}$ spherical end of a radial resin canal, and scale bar $=100 \mu \mathrm{m}$. 

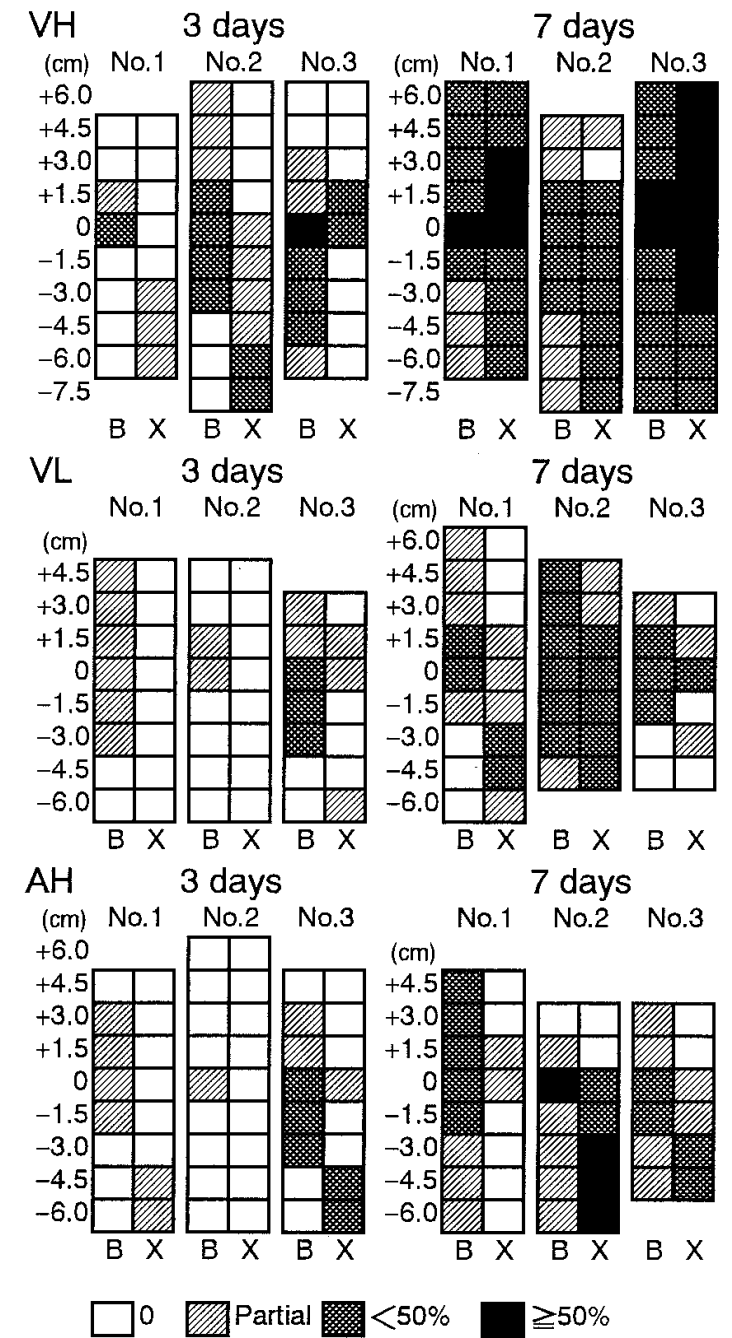

Fig. 4. Occurrence of disease symptoms in Pinus thunbergii seedlings inoculated with a virulent isolate of Bursaphelenchus xylophilus at high temperatures $\left(30\right.$ to $25^{\circ} \mathrm{C}$; $\left.\mathrm{VH}\right)$, a virulent isolate of pinewood nematode (PWN) at low temperatures $\left(25\right.$ to $20^{\circ} \mathrm{C}$; VL), and an avirulent isolate of PWN at high temperatures ( $\mathrm{AH})$ at 3 and 7 days after inoculation. Numbers above columns indicate seedling number. Each column indicates the discolored area in bark (B) and the area of cavitation in xylem $(\mathrm{X})$. Numbers at left side show the distance above $(+)$ and below $(-)$ the inoculation site in the stem of the seedling.

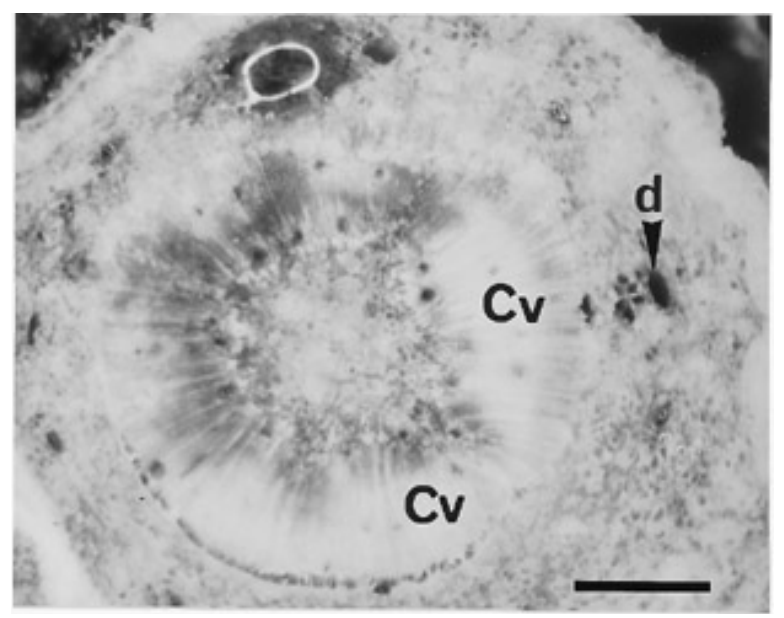

Fig. 5. Photomicrograph showing the area of cavitation $(\mathrm{Cv})$ in xylem and discolored cortical tissue (d) on a cut surface of a shoot at the inoculation site 3 days after inoculation of a Pinus thunbergii seedling with a virulent isolate of Bursaphelenchus xylophilus at high temperatures ( 30 to $25^{\circ} \mathrm{C}$ ). Scale bar $=1 \mathrm{~mm}$. distributed mainly in cortical resin canals 3 days after inoculation (Fig. 2). The number of PWN fragments in cortical resin canals was similar to the number in the VH treatment. PWN distribution in cortical tissue was limited to the inoculation site, compared with that of the $\mathrm{VH}$ treatment. The number of PWN fragments in xylem resin canals was less than that in the VH treatment. This became more evident at 7 days after inoculation; that is, the number of PWN was much less than that of the VH treatment, except in the cortical resin canals and other tissues around the inoculation site.

The restricted distribution of the virulent isolate of PWN at low temperatures $(3,4,11)$ and the avirulent isolate of PWN C14-5 $(2,5,6,7)$ has been observed by others by extraction of the PWN from tissues. Nematode extraction in this study showed that, in the VL and $\mathrm{AH}$ treatments, the distribution of PWN was restricted in bark tissue. By the histological observation, it was determined that, in the VL and $\mathrm{AH}$ treatments, PWNs were distributed in the cortical resin canals and invaded the cortical tissue only around the inoculation site. In contrast, a large population of PWNs in the $\mathrm{VH}$ treatment widely invaded cortical tissue from cortical resin canals breaking cell walls. These differences suggest that the ability of PWNs to invade cortical tissue may be stronger at high temperatures than at low temperatures, and that the ability of the virulent isolate may be stronger than that of the avirulent isolate. One possible cause of this difference could be the ability of PWN to produce cellulase $(24,28)$, the activity of which is stronger in virulent than in avirulent isolates (15). The destruction of pine cells and tissues has been observed after PWN inoculaton (1619,21,23).

In the xylem, the number of PWNs in the $\mathrm{VH}$ treatment was larger than that in the $\mathrm{AH}$ and VL treatments. PWNs do not need to break cell walls to migrate in xylem resin canals and, at the inoculation site, PWNs entered the xylem resin canals directly through the wounds. Therefore, fewer nematodes in the xylem in the $\mathrm{AH}$ and VL treatments might be the result of the lower ability to migrate and multiply in xylem resin canals. Avirulent isolates of PWN have a lower ability to migrate and multiply than do virulent isolates of PWN $(2,5-7,9,13)$, and virulent isolates at low temperatures have a lower ability to multiply than at high temperatures $(1,3,4,11)$.

The observations of cut surfaces showed that discoloration occurred in cortical resin canals and cortical tissue near the inoculation site and partial cavitation occurred in the xylem within 3 days after inoculation in the VH treatment (Figs. 4 and 5). Discoloration in the cortex and cavitation in the xylem increased in infected tissues 7 days after inoculation. In the VL and $\mathrm{AH}$ treatments, the discoloration of the cortical 


\section{$\mathrm{VH}$}

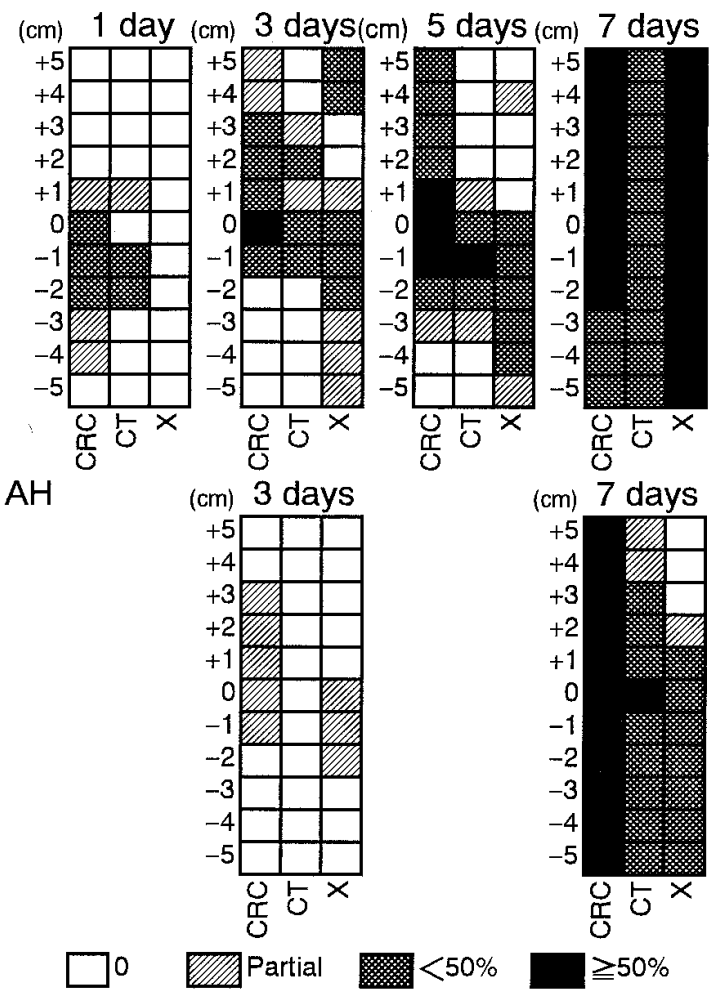

Fig. 6. Occurrence of disease symptoms in Pinus thunbergii seedlings inoculated with a virulent isolate of Bursaphelenchus xylophilus at high temperatures $\left(30\right.$ to $\left.25^{\circ} \mathrm{C} ; \mathrm{VH}\right)$ at $1,3,5$, and 7 days and an avirulent isolate of PWN at high temperatures (AH) at 3 and 7 days after inoculation. Each column indicates discolored area in cortical resin canals (CRC) and cortical tissue (CT) and area of cavitation in xylem (X). Numbers at left side show the distance above (+) and below (-) the inoculation site in the stem of the seedling. resin canals was on almost every cut surface 3 days after inoculation and visible in cortical tissue 7 days after inoculation (Fig. 4). However, cavitation in the xylem rarely occurred in either the VL or $\mathrm{AH}$ at 3 days, and was not as widespread 7 days after inoculation as in the $\mathrm{VH}$ treatment (Fig. 4).

These results indicate that, as the PWN number increased, so did disease symptom severity in the infected tissues. In the xylem, severe cavitation occurred in the $\mathrm{VH}$ treatment, whereas less-severe cavitation occurred in the VL and $\mathrm{AH}$ treatments, in which smaller numbers of PWNs were distributed. In cortical tissue, less-severe bark discoloration occurred in the VL and AH treatments, in which PWNs were restricted in cortical resin canals. Similar results have been reported $(2,25)$, in which an avirulent isolate of PWN or the less pathogenic nematode, $B$. mucronatus Mamiya and Enda, developed smaller populations in the xylem, so that the severity of cavitation was less than that of a virulent isolate of $\mathrm{PWN}$.

In the seedlings examined using the DAPI method, 1 day after inoculation in the $\mathrm{VH}$ treatment, small areas surrounding the cortical resin canals were discolored (Fig. 6). Discoloration of the cortical tissue increased to just less than $50 \%$ of cut surfaces at 3 days after inoculation. By 7 days after inoculation, discoloration was more apparent in the cortical tissues. Parenchy-
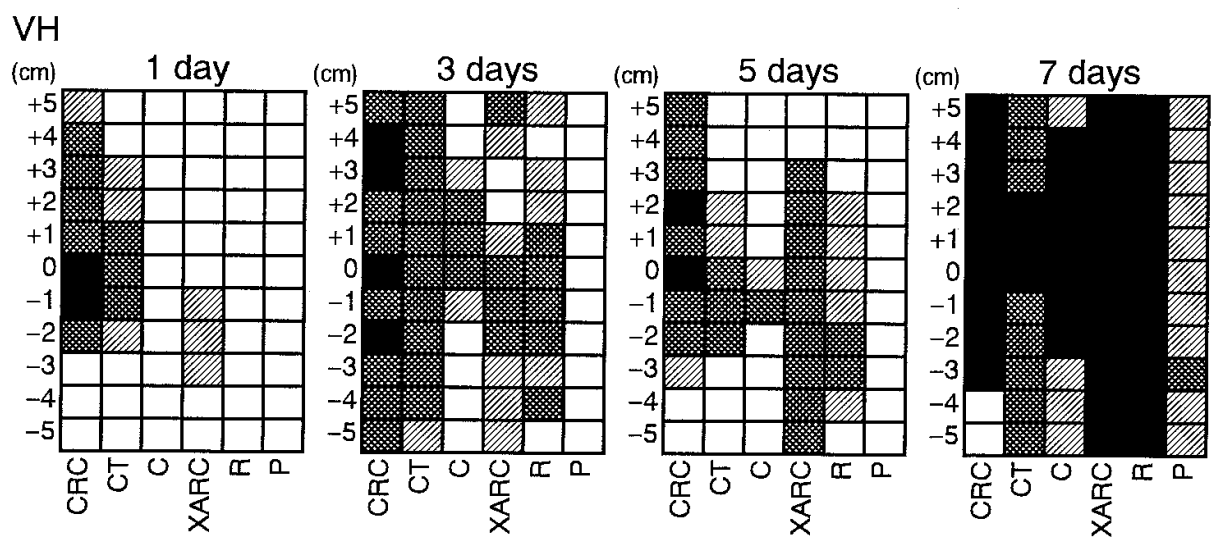

$\mathrm{AH}$
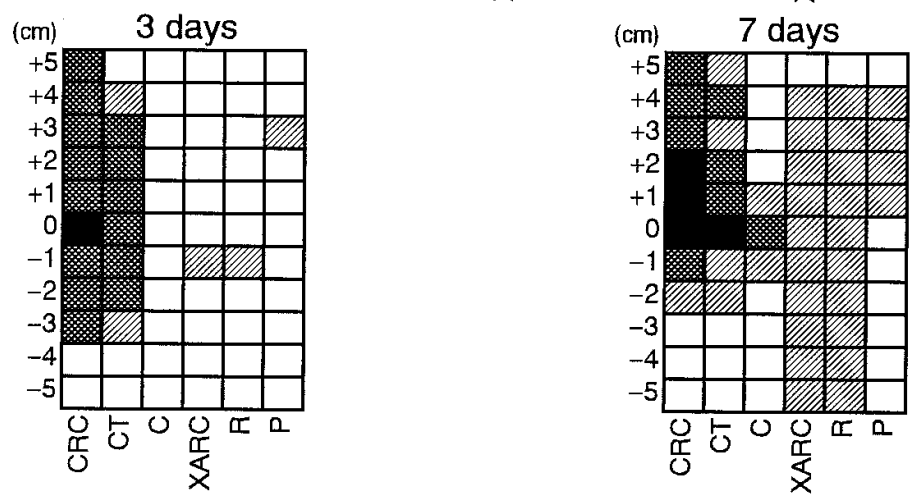

Fig. 7. Distribution of dead cells in Pinus thunbergii seedlings inoculated with a virulent isolate of Bursaphelenchus xylophilus at high temperatures (30 to $\left.25^{\circ} \mathrm{C} ; \mathrm{VH}\right)$ at $1,3,5$, and 7 days and an avirulent isolate of pinewood nematode at high temperatures $(\mathrm{AH})$ at 3 and 7 days after inoculation. Each column indicates the area of dead cells in cortical resin canals (CRC), cortical tissue (CT), cambial zone (C), axial resin canal in xylem (XARC), ray (R), and pith (P). Numbers at left side show the distance above (+) and below (-) the inoculation site in the stem of the seedling. 
matous cells appeared alive where no disease symptoms occurred, and cell death occurred where cortical tissue was discolored (Fig. 7). In the xylem, no cavitation occurred 1 day after inoculation (Fig. 6), but epithelial cells appeared collapsed and dead in parts of xylem resin canals (Fig. 7). Cavitation around xylem resin canals occurred within 3 days and continued until 5 days after inoculation. At the same time, dead cells were observed in axial resin canals in the xylem. At 7 days after inoculation, the area of cavitation enlarged to more than $50 \%$ of cut surfaces. More than $50 \%$ of parenchyma cells in the xylem appeared dead at that time.

In the $\mathrm{AH}$ treatment, discoloration of the cortical resin canals occurred around the inoculation site but no disease symptoms occurred in the cortical tissue 3 days after inoculation (Fig. 6). Most of the cortical resin canals were discolored and some of the surrounding cortical tissue was partially discolored in all cut surfaces at 7 days after inoculation, as in the VH treatment. However, in the xylem, cavitation occurred only around resin canals 7 days after inoculation (Fig. 6). Cell death occurred in parts of the discolored cortical tissue but seldom in the xylem resin canals until 7 days after inoculation (Fig. 7).

These results demonstrated that the area of discoloration in bark and cavitation in the xylem appeared to coincide with the area where parenchymatous cell death occurred. Disease symptoms and cell death occurred in both xylem and bark with a virulent isolate. In contrast, disease symptoms occurred only in cortical resin canals and rarely in the cortical tissue and xylem with an avirulent isolate. These close relationships also have been reported between discoloration and cell death in the bark $(8,9)$, and between cavitation and cell death in the xylem (6). It was shown that cell death and early symptom development coincided with PWN migration; therefore, PWN invasion induces cell death and early symptom development. PWN migration is an important factor in increasing the severity of disease symptoms in infected pine trees.

\section{LITERATURE CITED}

1. Dozono, Y., and Yoshida, N. 1974. Application of the logistic curve for the population growth of pine wood nematode, Bursaphelenchus lignicolus, on the cultures of Botrytis cinerea. J. Jpn. For. Soc. 56:146-148.

2. Fukuda, K., Hogetsu, T., and Suzuki, K. 1992. Cavitation and cytological changes in xylem of pine seedlings inoculated with virulent and avirulent isolates of Bursaphelenchus xylophilus and B. mucronatus. J. Jpn. For. Soc. 74:289-299.

3. Hashimoto, H., and Chihara, K. 1976. Pathological study of the pine wilting disease caused by Bursaphelenchus lignicolus under different conditions of temperature. Trans. Jpn. For. Soc. 87:237-238. (In Japanese)

4. Hotta, T., Masuda, T., and Hashimoto, H. 1975. Effect of temperature on the pathogenicity of Bursaphelenchus lignicolus. Trans. Jpn. For. Soc. 86:303-304. (In Japanese)

5. Ikeda, T. 1996. Responses of water-stressed Pinus thunbergii to inoculation with avirulent pine wood nematode (Bursaphelenchus xylophilus): water relation and xylem histology. J. For. Res. 1:223-226.

6. Ikeda, T., and Kiyohara, T. 1995. Water relation, xylem embolism and histological features of Pinus thunbergii inoculated with virulent or avirulent pine wood nematode, Bursaphelenchus xylophilus. J. Exp. Bot. 46:441-449.

7. Ikeda, T., Kiyohara, T., and Kusunoki, M. 1990. Change in water status of Pinus thunbergii Parl. inoculated with species of Bursaphelenchus. J. Nematol. 22:132-135.

8. Ishida, K., and Hogetsu, T. 1997. Role of resin canals in the early stages of pine wilt disease. Can. J. Bot. 75:346-351.

9. Ishida, K., Hogetsu, T., Fukuda, K., and Suzuki, K. 1993. Cortical responses in Japanese black pine to attack by the pine wood nematode. Can. J. Bot. 71:1399-1405.

10. Ishida, K., Suzuki, K., and Hogetsu, T. 1992. Spreading of nuclear degradation in Pinus thunbergii Parl. cuttings under water stress. Plant Cell Physiol. 33:897-907.

11. Kiyohara, T. 1973. Effect of temperature on the disease incidence of pine seedlings inoculated with Bursaphelenchus lignicolus. Trans. Jpn. For. Soc. 84:334-335. (In Japanese)

12. Kiyohara, T. 1984. Pine wilt resistance induced by prior inoculation with avirulent isolate of Bursaphelenchus xylophilus. The resistance mechanisms of pine against pine wilt disease. Pages 178-184 in: Proc. U.S. Jpn. Semin. Honolulu, Hawaii. V. Dropkin, ed. National Science Foundation, Washington, D.C.

13. Kiyohara, T. 1989. Etiological study of pine wilt disease. Bull. For. For. Prod. Res. Inst. 353:127-176. (In Japanese, English summary)

14. Kiyohara, T., and Bolla, R. I. 1990. Pathogenic variability among populations of the pinewood nematode, Bursaphelenchus xylophilus. For. Sci. 36:1061-1076.
15. Kojima, K., Kamijyo, A., Masumori, M., and Sasaki, S. 1994. Cellullase activities of pinewood nematode isolates with different virulence. J. Jpn. For. Soc. 76:258-262. (In Japanese, English summary)

16. Kuroda, K., and Mamiya, Y. 1986. Behavior of the pine wood nematode in pine seedlings growing under aseptic conditions. Trans. Jpn. For. Soc. 97:471-472. (In Japanese)

17. Kusunoki, M. 1987. Symptom development of pine wilt disease-Histopathological observations with electron microscopes. Ann. Phytopathol. Soc. Jpn. 53:622-629.

18. Mamiya, Y. 1975. Behavior of Bursaphelenchus lignicolus in the wood of pine seedlings and pathological responses of pine to nematode infection. Trans. Jpn. For. Soc. 86:285-286. (In Japanese)

19. Mamiya, Y. 1980. Inoculation of the first year pine (Pinus densiflora) seedlings with Bursaphelenchus lignicolus and the histopathology of diseased seedlings. J. Jpn. For. Soc. 62:176-183. (In Japanese, English summary)

20. Mamiya, Y. 1983. Pathology of the pine wilt disease caused by Bursaphelenchus xylophilus. Annu. Rev. Phytopathol. 21:201-210.

21. Mamiya, Y. 1985. Initial pathological changes and disease development in pine trees induced by the pine wood nematode, Bursaphelenchus xylophilus. Ann. Phytopathol. Soc. Jpn. 51:546-555.

22. Melakeberhan, H., Rutherford, T. A., and Webster, J. M. 1992. Influence of temperature on reproduction of Bursaphelenchus xylophilus and Pinus sylvestris mortality. Nematologica 38:80-87.

23. Myers, R. F. 1986. Cambium destruction in conifers caused by pinewood nematodes. J. Nematol. 18:398-402.

24. Odani, K., Sasaki, S., Nishiyama, Y., and Yamamoto, N. 1985. Early symptom of pine wilt disease by hydrolytic enzymes produced by pine wood nematodes-Cellulase as a possible candidate of the pathogen. J. Jpn. For. Soc. 67:366-372.

25. Odani, K., Sasaki, S., Yamamoto, N., Nishiyama, Y., and Tamura, H. 1985. Differences in dispersal and multiplication of two associated nematodes, Bursaphelenchus xylophilus and Bursaphelenchus mucronatus in pine seedlings in relation to the pine wilt disease development. J. Jpn. For. Soc. 67:398 403.

26. Sasaki, S., Odani, K., Nishiyama, Y., and Hayashi, Y. 1984. Development and recovery of pine wilt disease studied by tracing ascending sap flow marked with water soluble stains. J. Jpn. For. Soc. 66:141-148.

27. Thorne, G. 1961. Principles of Nematology. McGraw-Hill, New York.

28. Yamamoto, N., Odani, K., Sasaki, S., and Nishiyama, Y. 1986. Cellulase exudation by the pine wood nematode-Detection of activity in its crawling track. J. Jpn. For. Soc. 68:237-240. 